\title{
DESIGN AND ANALYSIS OF LOW VOLTAGE, LOW POWER FULLY RECYCLING FOLDED CASCODE AMPLIFIER
}

\author{
Mounika Katta ${ }^{1} \&$ srinivas $E^{2}$
}

Abstract- In this paper design of low voltage low power A fully recycling folded cascode amplifier is designed mostly all trasistors are in subthreshold region which allowes low voltage with low power dissipatioon. The proposed amplifier designed using possitive feedback technique. The proposed amplifier gives good performance over that of the conventional folded cascode (FC) amplifier. This amplifier implemented in cadence virtuoso $180 \mathrm{~nm}$ CMOS technology and operated with a $600 \mathrm{mV}$ supply voltage, $900 \mathrm{fF}$ capacitor load. The proposed FRFC amplifier achieves $73.5 \mathrm{~dB}$ DC gain (73.5 dB versus $55 \mathrm{~dB})$ and better phase margin as 56 degrees. The amplifier consumes $7 \mu \mathrm{W}$ which make it suitable for low power bio medical applications.

Keywords- FRFC: Fully Recycling Folded Cascode, FC: Folded Cascode

\section{INTRODUCTION}

Operational Amplifier is an integral part of many analogue and mixed signal systems. An amplifier whose differential input voltage produces an output current. The design of amplifier continues to face challenge as the supply voltage and transistor length scale down with each newer generation of CMOS technologies. To address the issue of power efficiency, amplifier is used.

Operational amplifiers are extensively used in analog and mixed signal integrated circuits such as filters, data converters and many other applications. Among many amplifiers architectures, folded cascode amplifier is faster and give better transconductance response so it give more gain than the multi stage amplifiers. The input differential pair is the main part of operational amplifier. An amplifier means they can amplify signals in certain ratio of input to output. The ratio is commonly referred as the gain of the operational amplifier. They can be mostly used to amplify weak electrical current signals to strong electrical signals in radio, TV, and many other electrical devices..

In this paper, folded cascode amplifier with positive feedback technique is used to get good performance than the normal folded cascode amplifiers. These amplifiers are operated in $\pm 600 \mathrm{mv}$ of supply voltage so transistors of amplifiers are operated mostly in subthreshold region. Subthreshold or weak inversion region means gate to source voltage is below the threshold voltage. Subthreshold region have more importance now a days because due to subthreshold the supply voltage is scaled down continuously. In sub threshold region, MOSFET has low saturation voltages to get larger output voltage swing. For this subthreshold region we get the more gain and consumes less power than the active region. This amplifier consumes $<8 \mu \mathrm{W}$ of power and open loop gain $=73 \mathrm{~dB}$ is verified by the $0.18 \mu \mathrm{m}$ CMOS technology. .

This paper organized as follows. In Section II describes folded cascode structures those are folded cascode amplifier and recycling folded cascode amplifier. Section III describes proposed amplifier i.e., fully recycling folded cascode amplifier. Section IV demonstrates simulation results and discussions. Section V shows comparative analysis with conventional ones. Finally conclusion is presented.

\section{FOLDED CASCODE AMPLIFIERS}

Folded cascode architecture is used for high swing and high gain with low power consumption. This op-amp uses cascodeing in the output stage. It exhibits good gain and output voltage swing, slew rate, power consumption than the two stage operational amplifier. Folded cascode amplifier has more transconductance so the dc gain is more and the common mode rejection ratio is also high, and it is easier to stabilize, since single high impedance node at output stage. Fig 1 shows schematic of folded cascode amplifier.

\footnotetext{
${ }^{1}$ M.Tech Student, Anurag Group Of Institutions, Hyderabad, Telangana, India

${ }^{2}$ Associate Professor, Anurag Group Of Institutions, Hyderabad, Telangana, India
} 


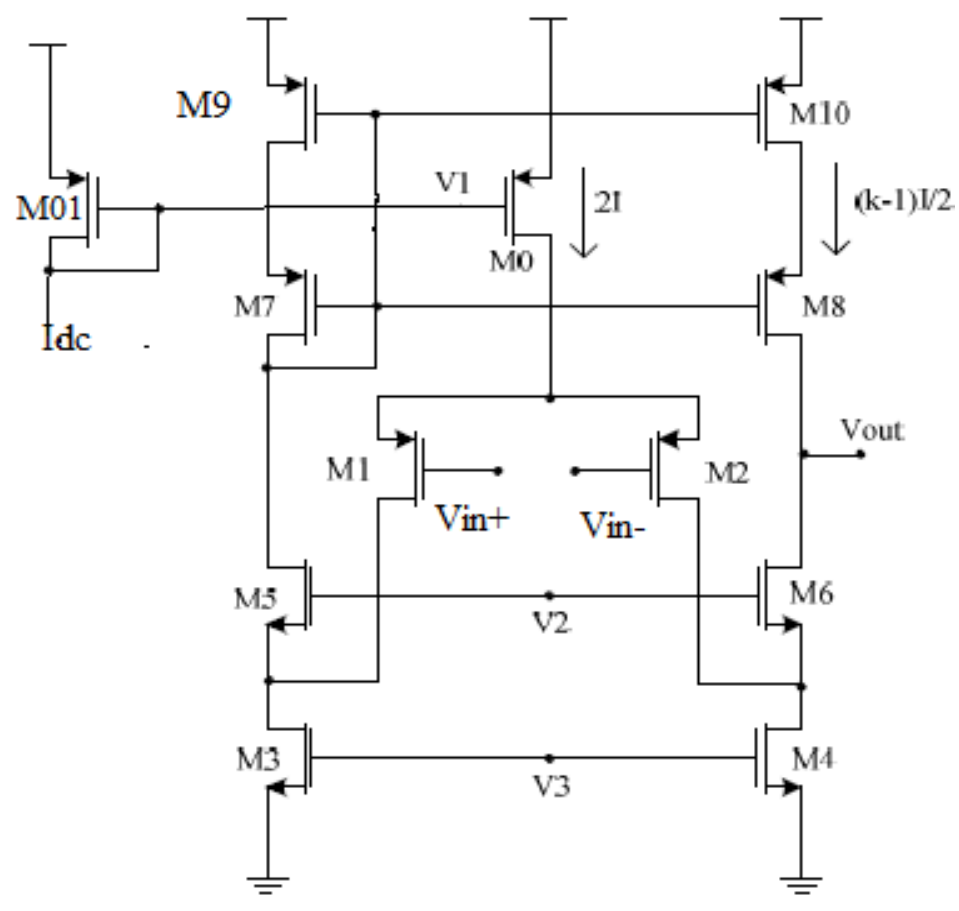

Fig 1: Folded cascode amplifier

In the above schematic the transistors M1,M2 are differential input pair. Current source is connected at M01, M0 transistors. M4, M5, M6, M7 transistors act as current mirror. Two bias voltages is given to M5, M6 and M3, M4.

Table 1: Input specifications

\begin{tabular}{|l|l|}
\hline & Value \\
\hline Vdd & $0.6 \mathrm{v}$ \\
\hline Vss & $-0.6 \mathrm{v}$ \\
\hline Unity gain frequency & $>3 \mathrm{MHz}$ \\
\hline Phase margin & $55-60 \mathrm{deg}$ \\
\hline Power consumption & $<10 \mu$ watts \\
\hline Slewrate & $>2 \mathrm{v} / \mu \mathrm{s}$ \\
\hline CMRR & $>100 \mathrm{~dB}$ \\
\hline Capacitor load & $900 \mathrm{fF}$ \\
\hline
\end{tabular}

\subsection{Recycling folded cascode amplifier}

In order to enhance folded cascode amplifier the RFC introduced, which has advantages of higher gain and better phase margin than conventional folded cascode amplifier the work on RFC used positive feedback to enhance performance of RFC. The transconductance is increased without consuming more power. In addition with the positive feedback can be used for increasing the output impedance which leads to higher DC gain. Input transistors of folded cascode i.e. M1,M2 are split in half to produce transistors M1a,M1b,M2a,M2b this will conducts fixed and equal currents. M3 and M4 are split to form current mirror M3a,M3b,M4a,M4b. The cross over connections of these current mirrors insures small signal currents added to source of M5 and M6 which are in phase. M11 and M12 are sized similar to M5 and M6 and the addition of it helps to maintain drain potentials of M3a and M3b \& M4a and M4b. The following schematic shows the recycling folder cascode amplifier. 


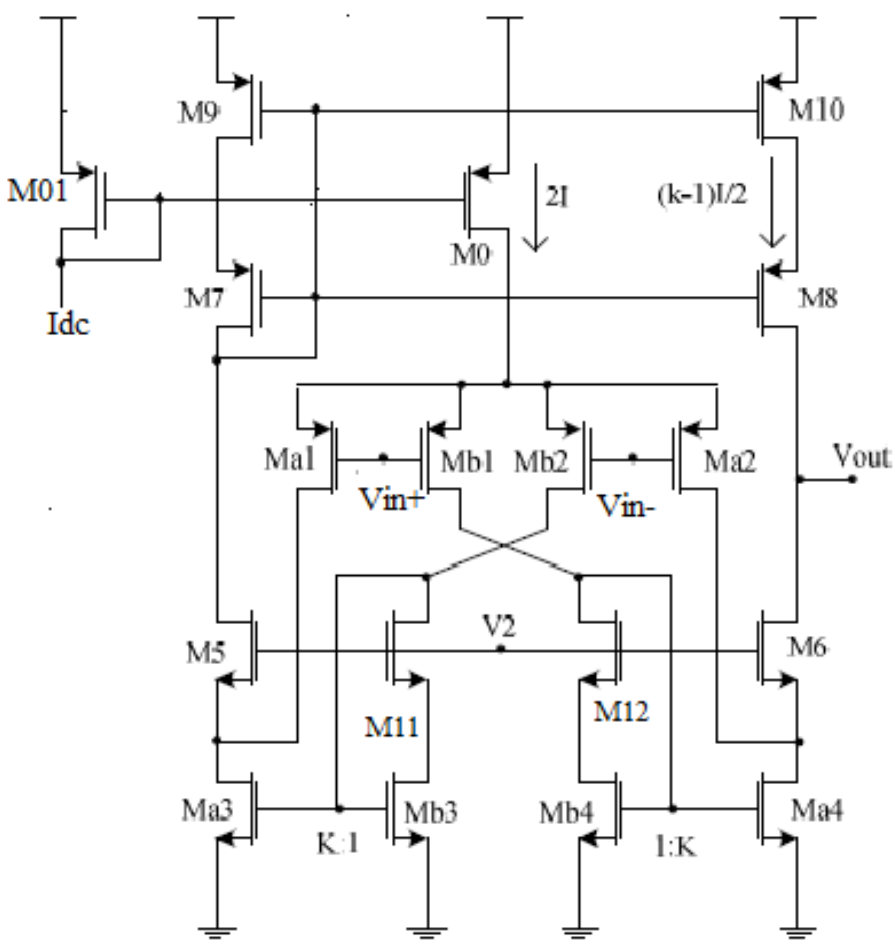

Figure2: Recycling Folded Cascode amplifier

\section{FULLY RECYCLING FOLDED CASCODE AMPLIFIER}

In FERFC M9\&M10 as driving transistors, a cross over connection between Mb3\& Mb4 with M9 and M10 established. To maintain amplifier as single ended architecture M11 and M12 are added. Ma3, Mc1, Mb3 act as high speed current mirrors in this a compensation resistor is used to maintain amplifier as more stable. The following schematic shows the fully recycling folded cascode amplifier. The amplifier's transconductance $(\mathrm{Gm})$ is found by calculating short-circuit current at the output with regards to the input. As mentioned previously, the M1 is the twice the size of Ma1 and conducts twice the amount of current $\left(\mathrm{gm}_{1}=2 \mathrm{gm}_{\mathrm{a} 1}\right)$.

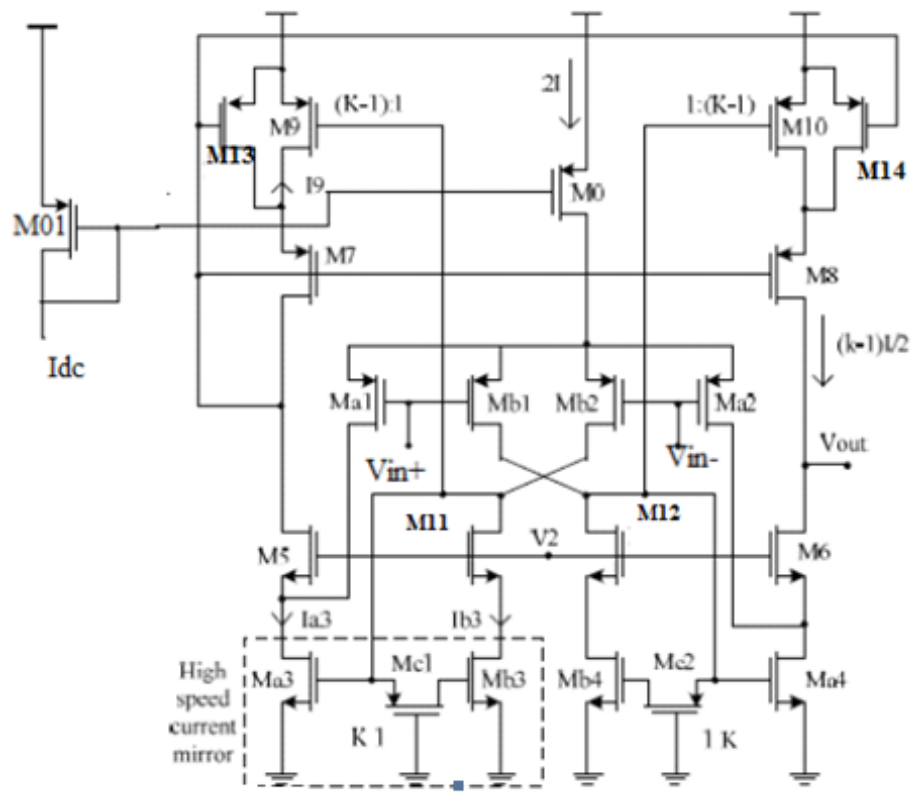

Figure3: Fully Recycling Folded Cascode Amplifier. 


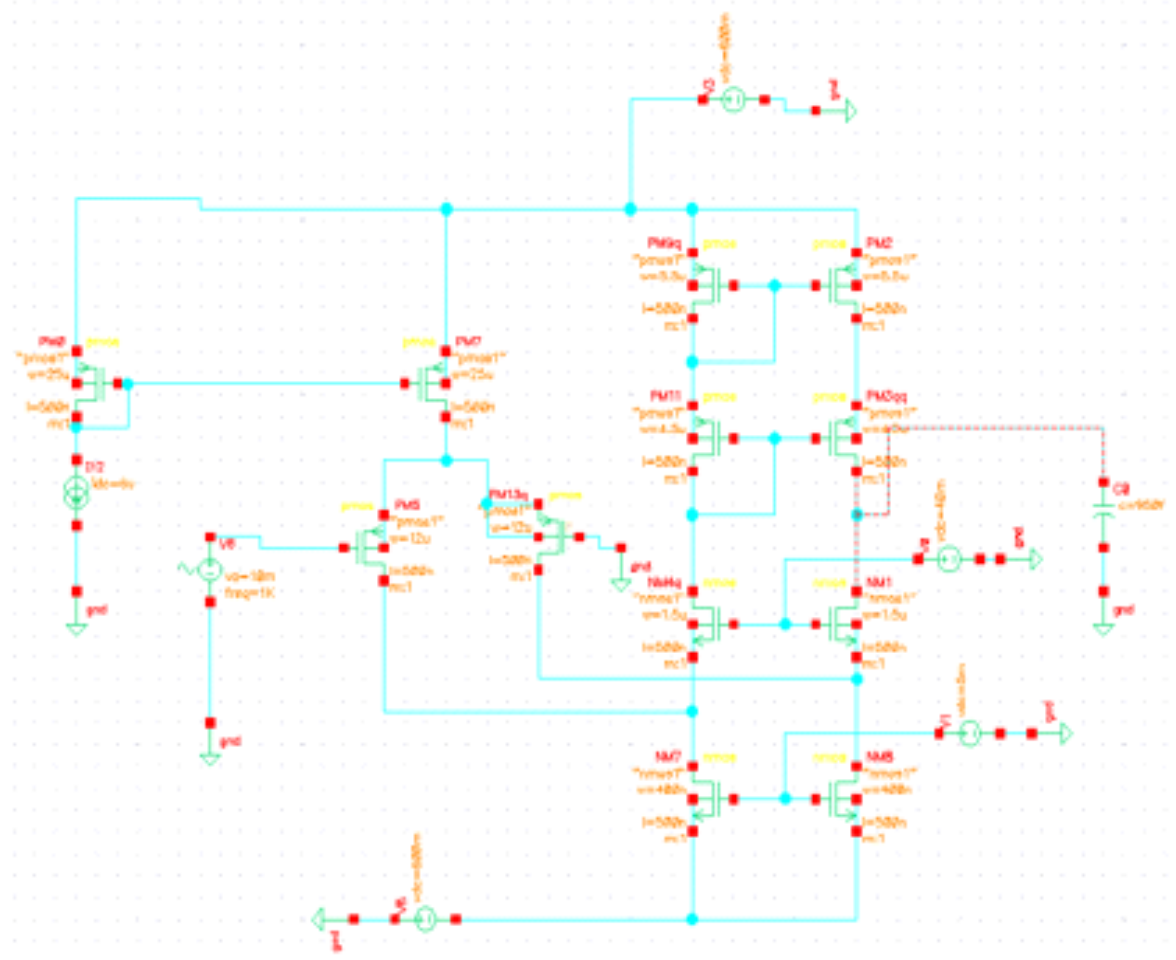

Figure4: Schematic of Folded Cascode Amplifier.

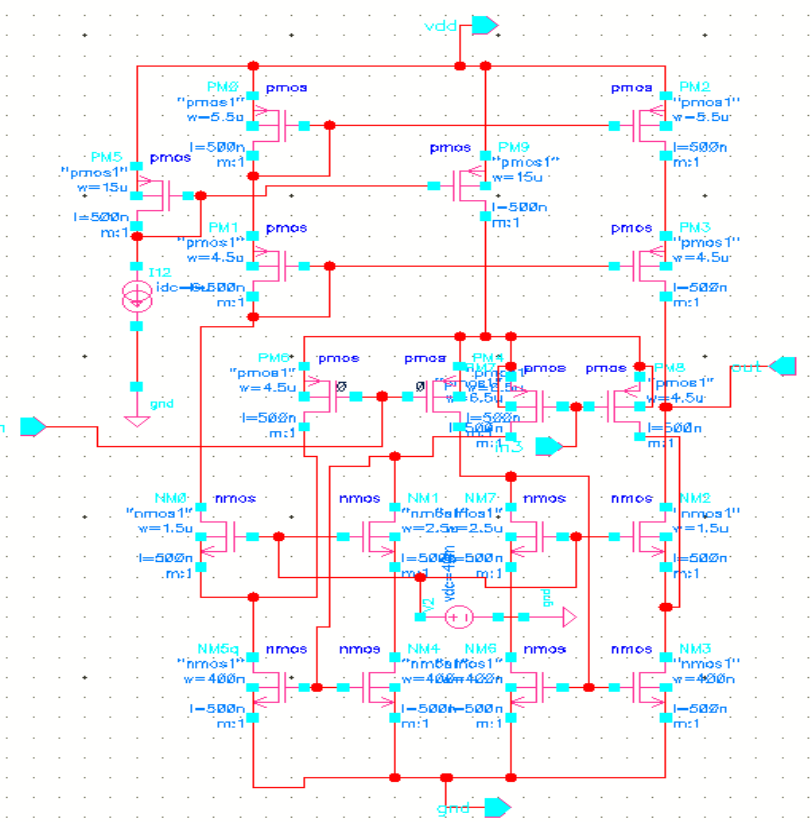

Figure5: Schematic of Folded Cascode Amplifier. 


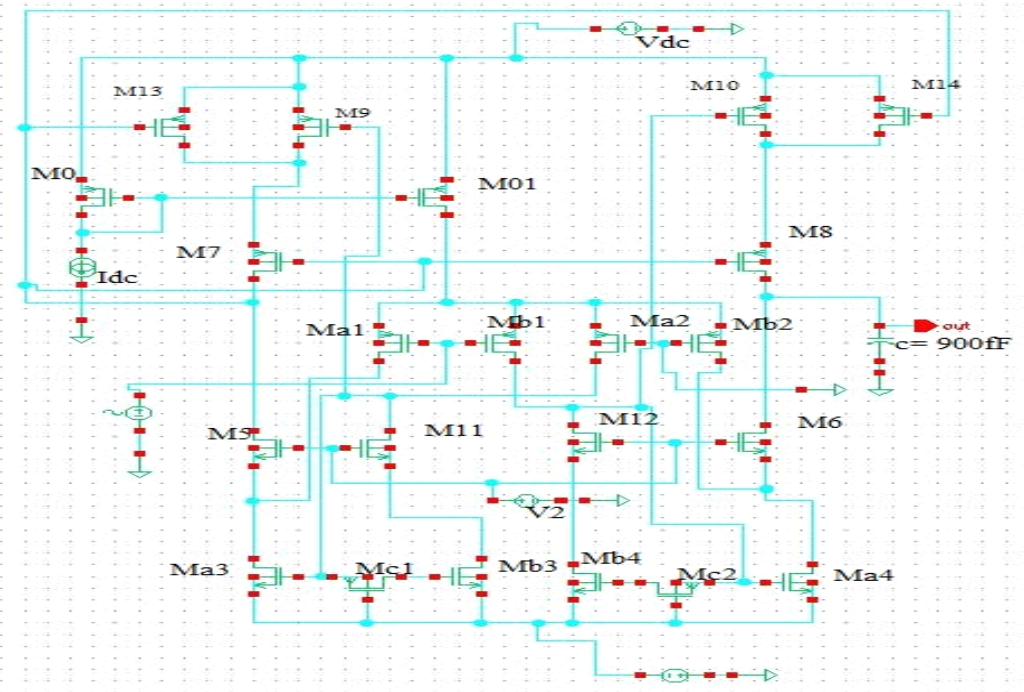

Figure 6: Schematic of Fully Recycling Folded cascode amplifier

In the above schematic sinusoidal signal applied as input signal to differential input pair. Current source is given as $6 \mu \mathrm{A}$. Transistors M1a, M1b, M2a, M2b introduce positive feedback and increase effective transconductance.

\section{RESULTS AND DISCUSION OF FULLY RECYCLING FOLDED CASCODE AMPLIFIER}

Simulation Results of the above circuits using Cadence Virtuoso simulator are

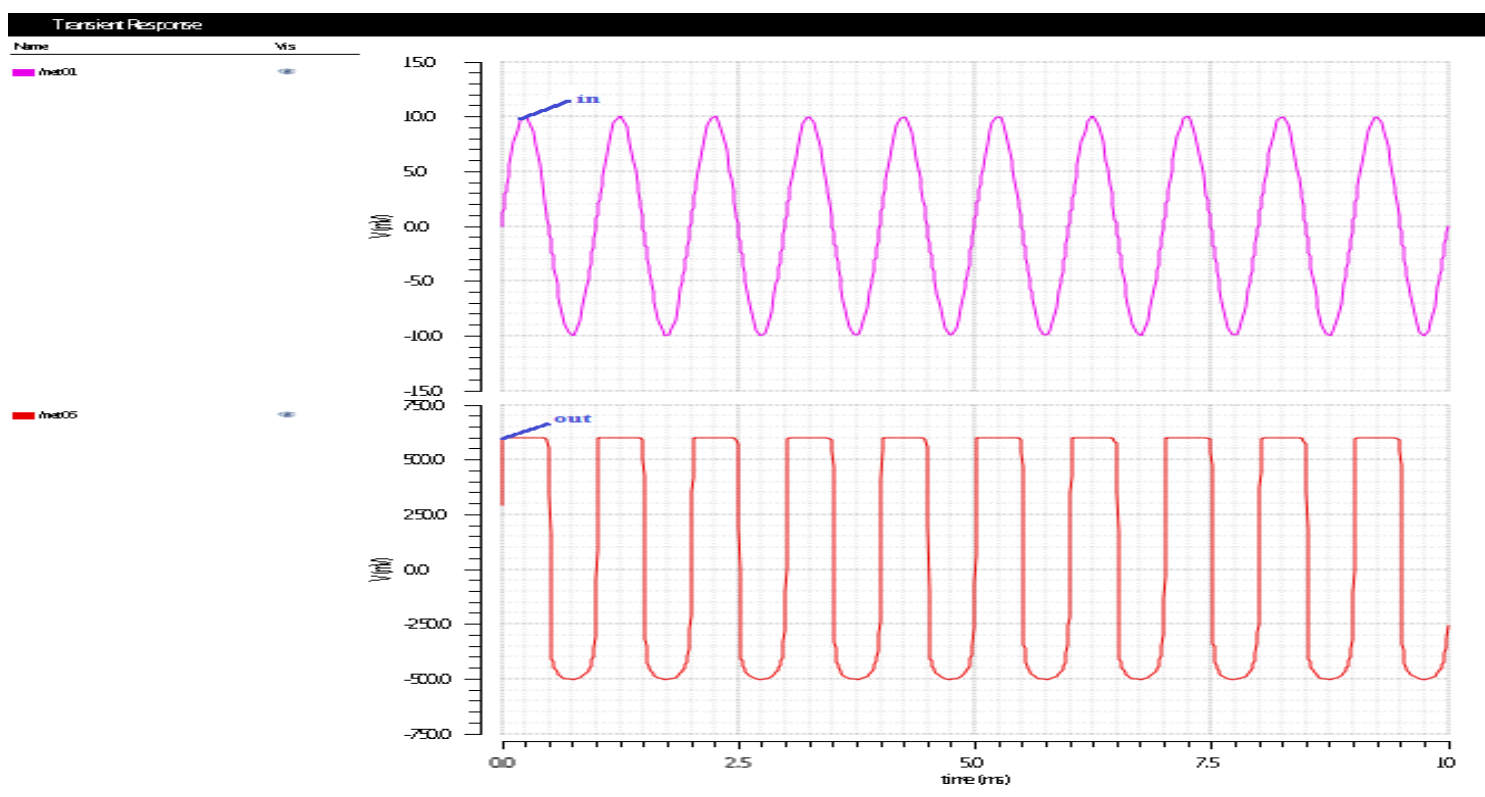

Figure 7:Transient response 


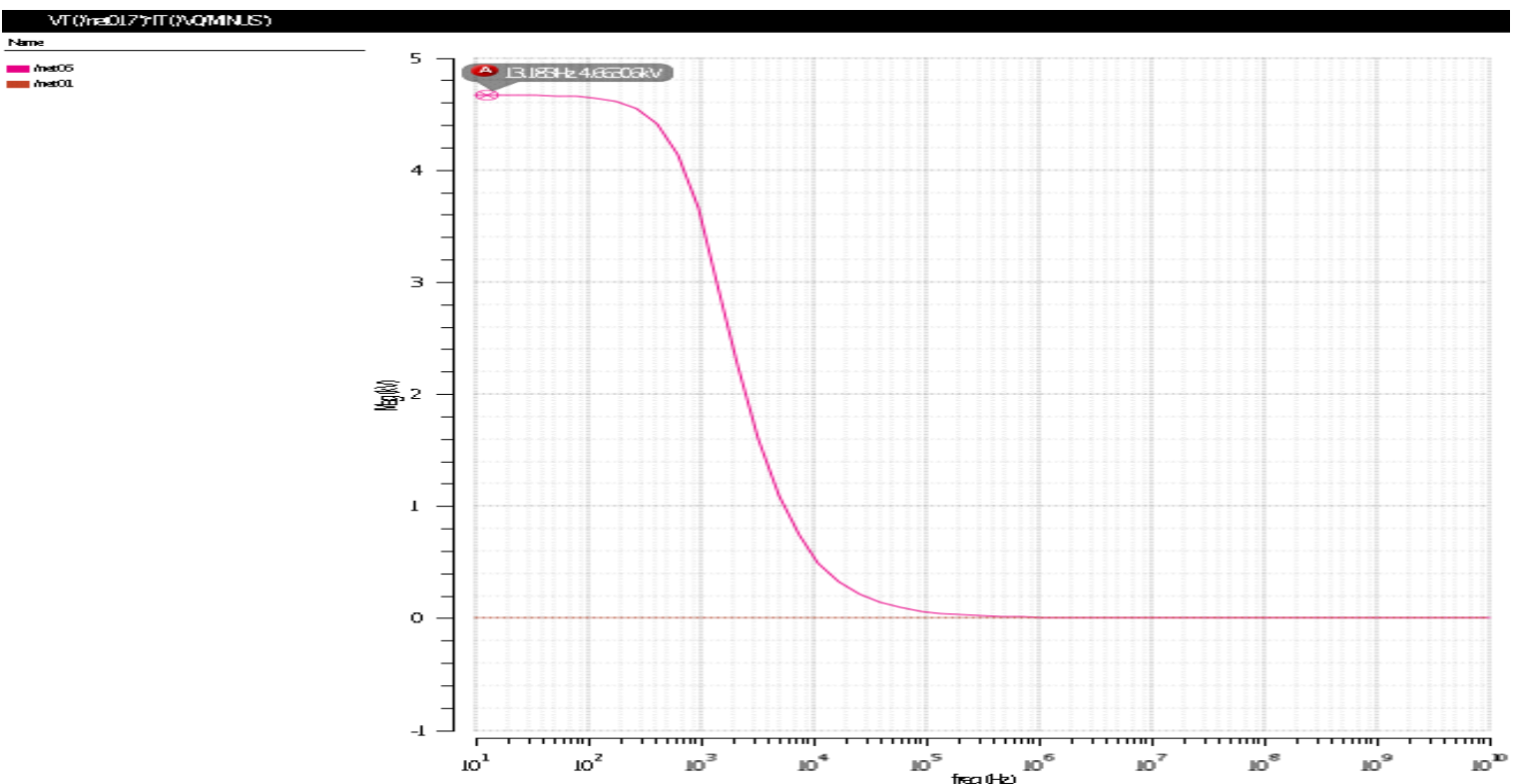

Figure 8:ACresponse

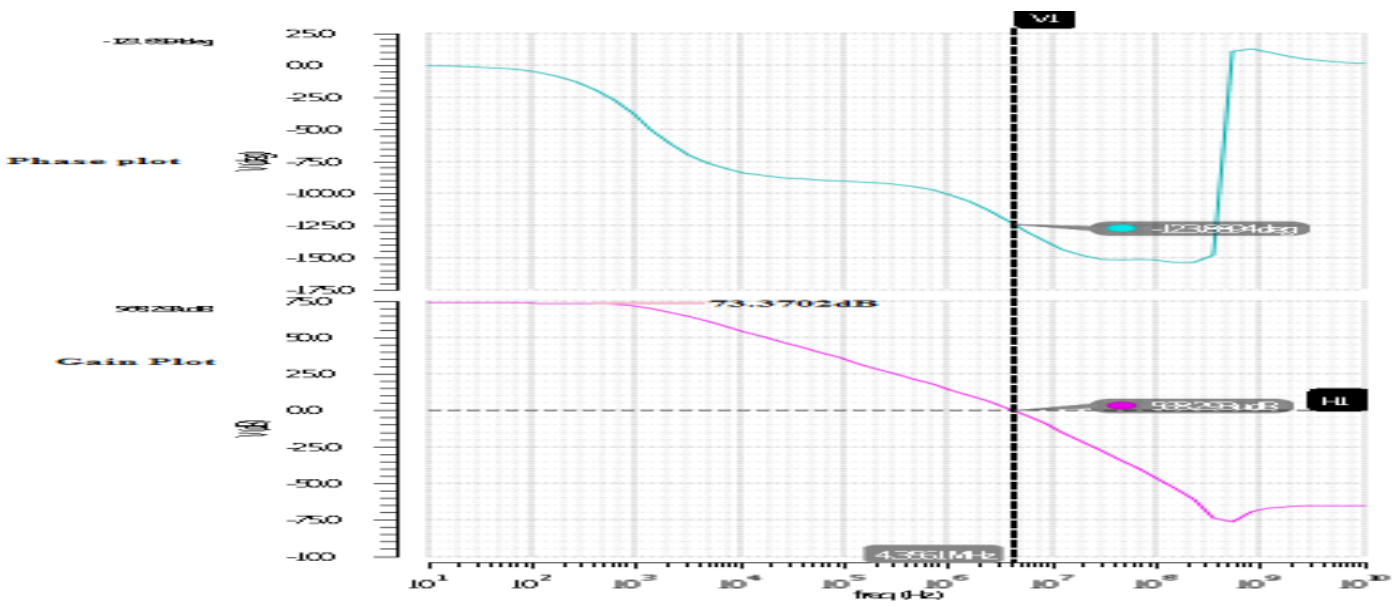

Figure 9: Gain And Phase response

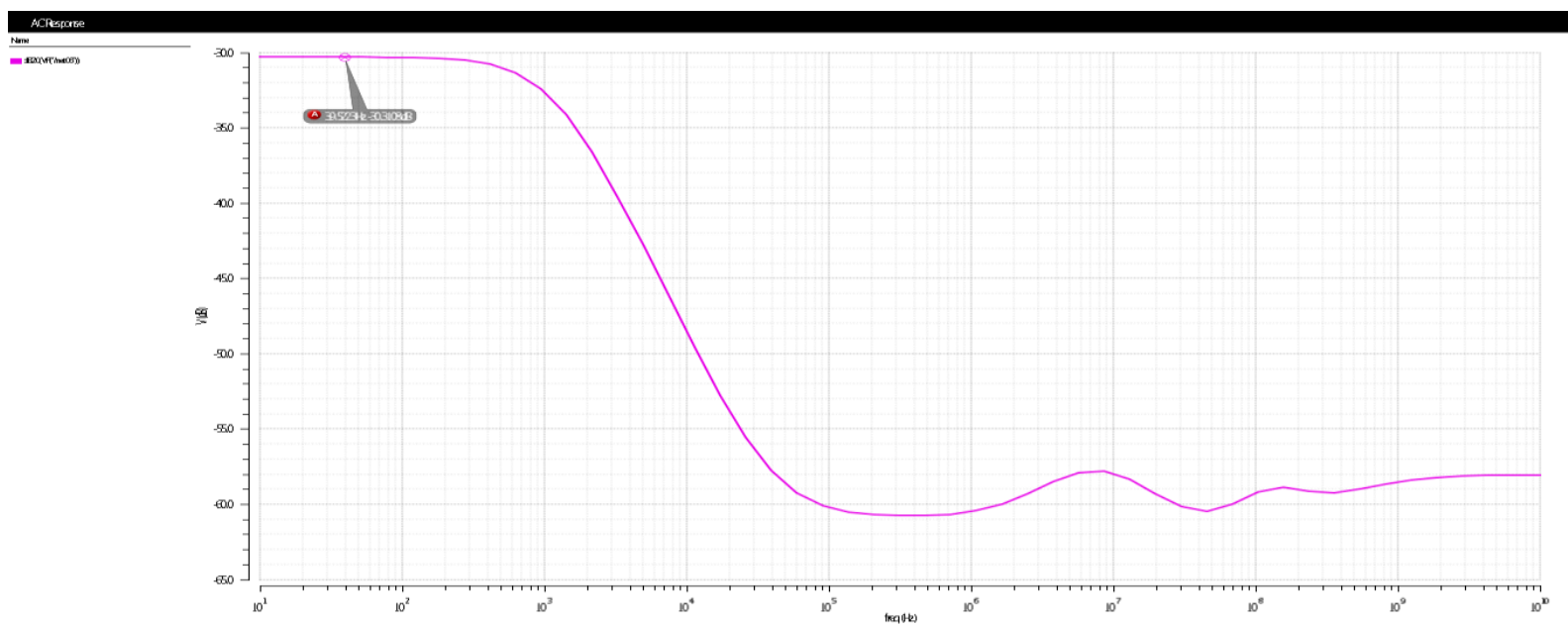

Figure 10: Common mode Gain 


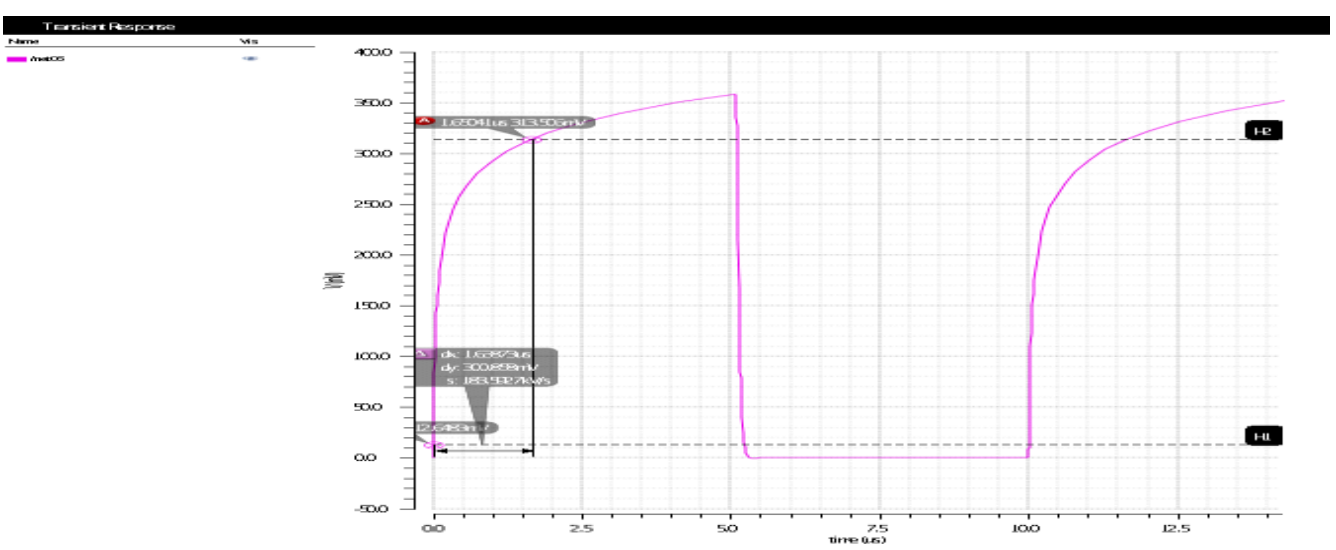

$\mathrm{SR}+\quad=183.68 \mathrm{~V} / \mathrm{ms}$

Figure 11: Positive Slew rate response

$=0.183 \mathrm{v} / \mu \mathrm{s}$

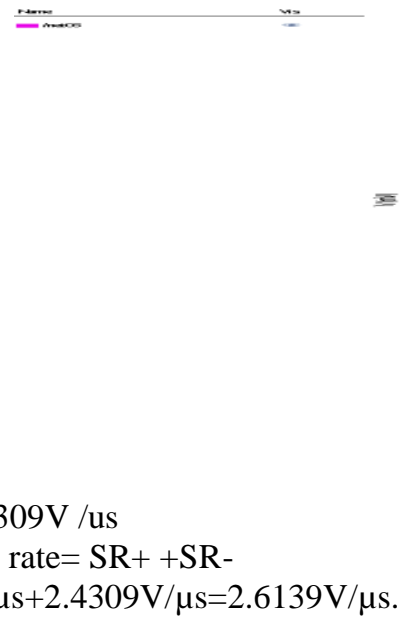

$\mathrm{SR}-=2.4309 \mathrm{~V} / \mathrm{us}$

$=0.183 \mathrm{v} / \mu \mathrm{s}+2.4309 \mathrm{~V} / \mu \mathrm{s}=2.6139 \mathrm{~V} / \mu \mathrm{s}$.

The below table shows the comparison of each parameter of proposed amplifier with the conventional amplifiers. Itshowsthat proposed amplifier gives Gain of $73 \mathrm{~dB}$ withhigh CMRR and better slew rate compared to the conventional Folded cascode amplifier. The proposed amplifier consumes less power compared with conventional amplifier

The creation of mask layout is one of the important steps in the full custom design flow, where the designer describes the detailed geometry and the relative positioning of each mask layer to be used in actual fabrication, using a layout editor.

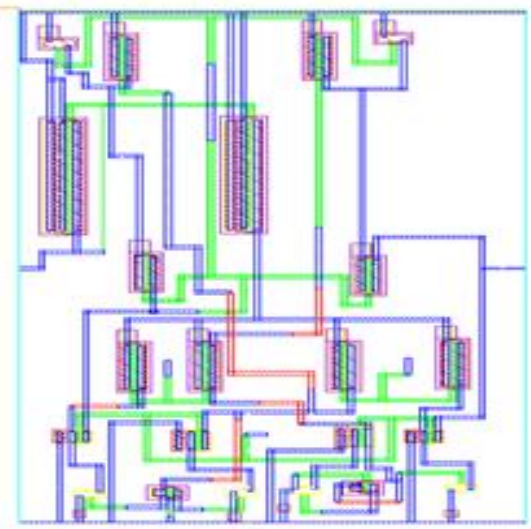

Figure13: Layout of Proposed Amplifier.

Layout must be drawn according to strict design rules. After you have finished our layout, an automatic program will check each and every polygon in the design against these design rules and report violations. This process is called Design Rule Checking and must be done for every layout to ensure that it will function properly when fabricated. The next step is to 
compare the net list extracted from the layout with the schematic to ensure that layout drawn is an identical match to the cell schematic.

\section{COMPARATIVE ANALYSIS AND CONCLUSION}

Table2: Comparative analysis of simulation results of folded cascode amplifiers

\begin{tabular}{|l|l|l|l|}
\hline Performances & Folded Cascode Amplifier & RFC Amplifier & Proposed Amplifier \\
\hline Technology $(\mathrm{nm})$ & 180 & 180 & 180 \\
\hline Power supply $(\mathrm{mV})$ & 600 & 600 & 600 \\
\hline Open loop gain $(\mathrm{dB})$ & 55 & 68.52 & 73.26 \\
\hline Phase Margin $($ degrees $)$ & 81.6 & 80 & 57 \\
\hline Unity gain bandwidth $(\mathrm{MHz})$ & 7.103 & 8.301 & 4.3227 \\
\hline CMRR $(\mathrm{dB})$ & 67.96 & 90.52 & 103.52 \\
\hline SR $(\mathrm{v} / \mu \mathrm{s})$ & 3.103 & 3.122 & 2.6139 \\
\hline PSRR+ $(\mathrm{dB})$ & 84.27 & 99.89 & 103.12 \\
\hline PSRR- $(\mathrm{dB})$ & 150.9 & 100.15 & 79.337 \\
\hline Power $(\mu \mathrm{W})$ & 7.443 & 7.443 & 6.99 \\
\hline
\end{tabular}

In this paper, The design of a low power and high gain CMOS Folded cascode amplifier is proposed, which can be used for bio-medical and low power applications. This design is carried out in CMOS 180nm technology, with the supply voltage of $600 \mathrm{mv}$. The circuit is operated in sub threshold region. The standard current mirror circuit is connected to make the current constant irrespective of the output load. This amplifier gives gain of about $73 \mathrm{~dB}$ with the effective CMRR of $103 \mathrm{~dB}$ and phase margin 56deg with low power consumption of $7 \mu$ watts.

\section{REFERENCES}

[1] R. S. Assaad and J. Silva-Martinez, "The Recycling Folded Cascode: A General Enhancement of the Folded Cascode Amplifier," solid-state circuits, IEEE J, vol. 44, pp. 2535-2542, 2009.

[2] X. Zhao, H. Fang, and J. Xu, "A transconductance enhanced recycling structure for folded cascode amplifier," Analog Integrated Circuits and Signal Processing, springer, vol. 72, pp. 259-263, 2012.

[3] Z. Yan, P.-I.Mak, and R. P. Martins, "Double recycling technique for folded-cascode OTA," Analog Integrated Circuits and Signal Processing, springer, vol. 71, pp. 137-141, 2012.

[4] Z. Xiao, F. Huajun, and X. Jun, "DC gain enhancement method for recycling folded cascode amplifier in deep submicron CMOS technology," IEICE electronics express, vol. 8, pp. 1450-1454, 2011.

[5] M. M. Amourah and R. L. Geiger, "Gain and bandwidth boosting techniques for high-speed operational amplifiers," in Circuits and Systems, 2001. ISCAS 2001.The 2001 IEEE International Symposium on, 2001, pp. 232-235 vol. 1.

[6] B.Razavi," Design of Analog CMOS Integrated Circuits”, New York: Mc-Graw Hill, 2001.

[7] D.Johns and Ken Martin “Analog Integrated Circuit Design”, Wiley India Pvt. Ltd, 1997.

[8] Z. Xiao, F. Huajun, and X. Jun, "Phase-margin enhancement technique for recycling folded cascode amplifier," Analog Integrated Circuits and Signal Processing, springer US, vol. 74, pp. 479-483, 2012. Bit CMOS Full-Adder Cell”, International Journal of Science and Research (IJSR).

[9] MeysamAkbari, OmidHashemipour, and Ardavanjavid," An ultra -low voltage, ultra -low power fully recycling folded cascode amplifier", the 22 nd Iranian conference on electrical engineering (ICEE 2014), May 20-22,2014, ShahidBeheshti university.

[10] David Solomon George1, Firoz Mon Y,'Design and Analysis of Improved Recycling Folded Cascode OTA with Gain and Phase Margin Enhancement",International Journal of Advanced Research in Electrical, Electronics and Instrumentation Engineering.

[11] T.V Prasula; D.Meganathan "Design and simmulation of low power, high bandwidth recycling folded cascode OTA", Fourth international conference on signal processing communication and networking (ICSCN).

[12] Po-Yu Kuo; Gang-Jhih Fan; Sheng-Da Tsai " The enhancement of recycling folded cascode amplifioer", 2016 IEEE International Conference on Consumer Electronics-Taiwan (ICCE-TW).

[13] Moaaz Ahmed; Ikramullah Shah: Fang Tang; Amine Bermak" An improved recycling folded cascode amplifier with gain boosting and phase margin enhancement" 2015 IEEE International Symposium on Circuits and Systems (ISCAS).

[14] PravanjanPatra; Pankaj Kumar Jha; AshudebDutta " An enhancement recycling folded cascode OTA with a positive feedback", 2013 IEEE Asia Pacific Conference on Postgraduate Reserch in Microelectronics (PrimeAsia).

[15] NordianaMukahar; SitiAishahCheKar "Improved recyclinfolded cascode OTA with current control circuit", 2012 IEEE Symposium on Humanities, Science and Engineering Reserch. 